\title{
Factors of Reflux Episodes With Post-reflux Swallow- induced Peristaltic Wave in Gastroesophageal Reflux Disease
}

\author{
Hairong Xu, ${ }^{1,2}$ Bixing Ye, ${ }^{1}$ Yu Ding, ${ }^{1}$ Meifeng Wang, ${ }^{1}$ Lin Lin, ${ }^{1}$ and Liuqin Jiang ${ }^{1 *}$ \\ ${ }^{I}$ Department of Gastroenterology, The First Affiliated Hospital of Nanjing Medical University, Nanjing, China; and ${ }^{2}$ Department of \\ Gastroenterology, The Third Affiliated Hospital of Soochow University, Changzhou, China
}

\section{Background/Aims}

It is known that post-reflux swallow-induced peristaltic wave (PSPW) index represents the chemical clearance of the esophagus. However, few studies have explored why some reflux episodes could induce PSPW while others in the same patient could not. The purpose of this study is to investigate the characteristics of reflux episodes which could elicit PSPW.

\section{Methods}

In this study, 269 reflux episodes were detected, of which 90 with a PSPW and 179 without a PSPW. Comparisons were made between the characteristics of reflux episodes with a PSPW and without a PSPW. The characteristics were including nadir $\mathrm{pH}, \mathrm{pH}$ drop, proximal extent $(\mathrm{cm}, \mathrm{sec})$, ascending velocity $(\mathrm{cm} / \mathrm{sec})$, volume clearance time, acid clearance time, percentage acidic $(\%), 15$ to 60-minute acid burden (seconds), and 15- to 60-minute volume burden (seconds). The characteristics between the 2 groups were compared through performing Wilcoxon signed rank test.

\section{Results}

Reflux episodes followed by a PSPW were significantly associated with a higher proximal extent than those without a PSPW. After the reflux episodes, higher volume clearance time and larger volume burden were more likely to trigger a PSPW. However, there were no significant differences between the 2 groups in nadir pH, pH drop, ascending velocity, acid clearance time, percentage acidic, or acid burden.

\section{Conclusions}

The role of acid seems to be less important in a reflux episode inducing a PSPW. Proximal reflux episodes are more likely to induce a PSPW. The depression of volume clearance may also be an important factor in eliciting a PSPW.

\section{(J Neurogastroenterol Motil 2020;26:378-383)}

\section{Key Words}

Esophageal pH monitoring; Gastric acid reflux; Gastroesophageal reflux

Received: May 24, 2019 Revised: September 18, 2019 Accepted: February 20, 2020

(.) This is an Open Access article distributed under the terms of the Creative Commons Attribution Non-Commercial License (http://creativecommons. org/licenses/by-nc/4.0) which permits unrestricted non-commercial use, distribution, and reproduction in any medium, provided the original work is properly cited.

*Correspondence: Liuqin Jiang, MD Department of Gastroenterology, The First Affiliated Hospital of Nanjing Medical University, 300\# Guangzhou Road, Gulou Strict, Nanjing 210029, China

Tel: +86-13951017379, E-mail: jiangliuqin@163.com

Hairong $\mathrm{Xu}$ and Bixing Ye contributed equally to this work. 


\section{Introduction}

Gastroesophageal reflux disease (GERD) is defined as a condition when the refluxed gastric contents causes troublesome symptoms and/or complications. ${ }^{1}$ Most patients can benefit from acid suppressant drugs. Twenty-four-hour multichannel intraluminal impedance and $\mathrm{pH}(\mathrm{MII}-\mathrm{pH})$ monitoring is a new and reproducible technique to monitor gastroesophageal reflux which has been reported to be highly sensitive for detection of all types of reflux events in the esophagus (gas, liquid, or mixed reflux episode). Moreover, 24-hour MII-pH monitoring is irrespective of acidity, so that it can also detect the reflux with a $\mathrm{pH}$ between 4 and 7 (weakly acidic reflux) or with a $\mathrm{pH}$ above 7 (alkaline reflux). ${ }^{2}$

Recently, a new defense mechanism of reflux events has been indicated which is known as post reflux swallow-induced peristaltic wave (PSPW). A PSPW was defined as an antegrade 50\% drop in impedance occurring within 30 seconds after a reflux event, originating in the most proximal impedance channel, reaching all distal impedance channels, and followed by at least $50 \%$ return to the baseline. ${ }^{3}$ The PSPW index was obtained when dividing the number of PSPWs by the number of reflux events. The PSPW index evaluates the efficacy of esophageal chemical clearance, which consists of a primary peristalsis and transit salivary bicarbonate and epidermal growth factor to the distal esophagus, thus helping repair of reflux-induced mucosal damage. Studies have showed that PSPW index is significantly lower in patients with reflux esophagitis and non-erosive reflux disease compared to controls and patients with functional heartburn. ${ }^{4}$ However, when analyzing the reflux we wondered why some reflux events will be followed by PSPWs while others are not. The purpose of this study is to investigate the characteristic of reflux episodes which could induce a PSPW.

\section{Materials and Methods}

\section{Subjects}

Ten patients were enrolled in this study, who were from the Gastrointestinal Motility Center of the First Affiliated Hospital of Nanjing Medical University, China. All patients had symptoms, lasting $>6$ months, and their reflux disease questionnaire scores were not less than $12 .^{5}$ The medical ethics committee of the Nanjing Medical University approved this protocol (2014-SR-010), and we obtained written informed consent from all subjects.

\section{Esophageal Multichannel Intraluminal Impedance and pH Monitoring}

All subjects underwent MII-pH monitoring off-therapy, that was, proton pump inhibitors, histamine $\mathrm{H}_{2}$ receptor antagonists, and drugs influencing esophageal motor function were discontinued at least 2 weeks prior to the test. An ambulatory MII-pH monitoring system (Given Imaging, Duluth, GA, USA) was used to measure esophageal intraluminal $\mathrm{pH}$ and impedance values. MII$\mathrm{pH}$ catheter was passed transnasally. The $\mathrm{pH}$ sensor was placed 5 $\mathrm{cm}$ above the lower esophageal sphincter (LES), and 6 impedance channels (z1, z2, z3, z4, z5, and z6) were placed at 6 sites $(17,15,9$, 7,5 , and $3 \mathrm{~cm}$ above the LES, respectively). The MII-pH parameters were measured automatically by the software of the monitoring system (Given Imaging). During the 24-hour when MII-pH test was performed, meal timings, timing of any symptoms, and changes in body position were recorded by pressing the button on the data recorder. These data were downloaded into a personal computer and automatically analyzed by the monitoring-system software. The above procedure was conventional routine according to reference and instructions of esophageal MII-pH monitoring. ${ }^{6}$

\section{Data Analysis}

The reflux episodes were conjointly analyzed by 2 experienced investigators who finally made a consensus decision together. When analyzing the data curve, the signals recorded at the time of eating and drinking were not included in the statistics.

In the curve analysis, the proximal extent was measured as the distance of the proximal sensor to the LES and the time required to reach the proximal sensor. In addition, the nadir $\mathrm{pH}$ of the reflux episode, the change in $\mathrm{pH}$ (which was calculated by the peak $\mathrm{pH}$ minus the nadir $\mathrm{pH}$ ) and the ascending velocity (which means the speed of the reflux episodes reaching the proximal sensor) were noted for each reflux episode. The volume clearance time was noted as the time in seconds from the $50 \%$ drop in the most distal baseline impedance to this impedance restored above this point again. ${ }^{7}$ The acid clearance time was considered as the time in seconds when the esophageal $\mathrm{pH}$ value went below 4 to recovery to 4 or till the start of a new reflux episode.

The acid burden was measured as the total time with $\mathrm{pH}$ value below 4 recorded in the 15-, 30-, 45-, and 60-minute time segment preceding the onset of each reflux episode. ${ }^{8}$ The volume burden was measured as the total reflux time (time from the $50 \%$ drop in the most distal baseline impedance until it was restored above the point) measured in the 15-, 30-, 45-, and 60-minute time segment preced- 
ing the onset of each reflux episode. All analyses, including the acid and volume burden measurements, excluded the 3 meals. These moments were ignored in the calculation of the acid burden and volume burden due to the short consumption time of the beverage.

\section{Statistical Methods}

Unless otherwise noted, data are presented as median (interquartile range $[\mathrm{IQR}]$ ). Wilcoxon signed rank test was used to compare the characteristics between reflux episodes with PSPW and without PSPW within subjects. A $P<0.05$ was considered statistically significant. Statistical analysis was performed using SPSS version 19.0 for windows (IBM Corp, Armonk, NY, USA).

\section{Results}

\section{Patient Population and Reflux Episodes}

Totally, 10 GERD patients were enrolled in this study who finished 24-hour $\mathrm{pH}$ and impedance recordings, including 4 males and 6 females aged 37-67 years old (median 53.9 years). The body mass index (median [IQR]) of the patients was 25.31 (22.70, $27.93) \mathrm{kg} / \mathrm{m}^{2}$. Forty percent $(4 / 10)$ of the patients were diagnosed of esophagitis by endoscopy. A total of 269 reflux episodes were detected and analyzed. Of all these episodes, 90 (33.46\%) were followed by PSPW. Acid exposure accounts for 62.01\% (111/179) of reflux episodes without PSPW, while 55.56\% (50/90) in reflux episodes with PSPW (Table 1).

\section{Acid and Volume Burden of Reflux Episodes With/ Without Post-reflux Swallow-induced Peristaltic Wave}

Reflux episodes with PSPW had longer time than reflux

Table 1. Clinical and Demographic Characteristics of the Study Population

\begin{tabular}{lc}
\hline \multicolumn{1}{c}{ Patient characteristics } & Data \\
\hline Total number of patients & 10 \\
Median age $(\mathrm{yr})$ & 53.9 \\
Female gender & $6(60.00)$ \\
BMI $\left(\mathrm{kg} / \mathrm{m}^{2}\right)$ & $25.31(22.70,27.93)$ \\
Non-erosive reflux disease & $6(60.00)$ \\
Total number of reflux episodes & 269 \\
Reflux with PSPW & $90(33.46)$ \\
Reflux without PSPW & $179(67.04)$ \\
\hline
\end{tabular}

BMI, body mass index; PSPW, post-reflux swallow-induced peristaltic wave. Data are presented as number (\%) or median (interquartile range). episodes without PSPW in 60-minute volume burden, (median [IQR]: $42.15[17.35,89.08]$ seconds vs $27.60[12.10,71.00]$ seconds; $P=0.027$ ). Longer time was also found in reflux episodes with PSPW than those without PSPW in other different times volume burden, but there was no statistically significant difference $(9.35$ $[0.00,27.18]$ seconds vs $2.1[0.00,18.90]$ seconds in 15 -minute volume burden, $P=0.936 ; 21.65[4.65,57.10]$ seconds vs 12.40 $[1.00,40.50]$ seconds in 30 -minute volume burden, $P=0.361$; $35.80[9.70,75.75]$ seconds vs $19.00[6.80,54.40]$ seconds in 45 -minute volume burden, $P=0.169$ ) (Fig. 1). No significant differences were found between these 2 groups in all different times acid burden: (11.40 [0.00, 72.90] seconds vs $0.00[0.00,85.70]$ seconds in 15 -minute volume burden, $P=0.058 ; 38.10$ [3.10, $190.10]$ seconds vs $32.30[0.00,178.30]$ seconds in 30 -minute volume burden, $P=0.798 ; 71.10[9.03,290.98]$ seconds vs 55.60 $[0.00,264.80]$ seconds in 45 -minute volume burden, $P=0.671$; $84.05[18.08,365.65]$ seconds vs $80.40[3.40,386.30]$ seconds in 60-minute volume burden, $P=0.693$ ) (Fig. 2).

\section{Characteristic Differences Between Reflux Episodes With Post-reflux Swallow-induced Peristaltic Wave and Reflux Episodes Without Post-reflux Swallow- induced Peristaltic Wave}

Reflux episodes with a PSPW were associated with a higher proximal extent than those without a PSPW $5.00[1.40,9.15] \mathrm{sec}-$ onds vs $3.70[1.00,6.80]$ seconds, $P=0.016)$. A higher volume clearance time before the start of the reflux episodes was found in reflux episodes with PSPW than those without PSPW (13.15 $[8.93,19.65]$ seconds vs $11.50[6.65,16.70]$ seconds, $P=0.029)$. However, no significant differences were found between the 2 groups in nadir $\mathrm{pH}, \mathrm{pH}$ drop, ascending velocity, acid clearance time, and percentage acidic (Table 2).

\section{Discussion}

GERD is considered as a significant public health problem globally. Recently, esophageal MII-pH monitoring has been widely studied to explore the underlying mechanisms of GERD. Defensive mechanisms of GERD include the anti-reflux barriers, principally the LES and the diaphragm, and luminal clearance mechanisms after an episode of reflux. ${ }^{9}$ Clearance of refluxate is biphasic which consists of volume clearance and chemical clearance. In the first phase, the rapid volume clearance removes the bulk of the refluxed bolus by peristalsis and gravity when positioned upright. In the second phase, chemical clearance restores the esophageal 

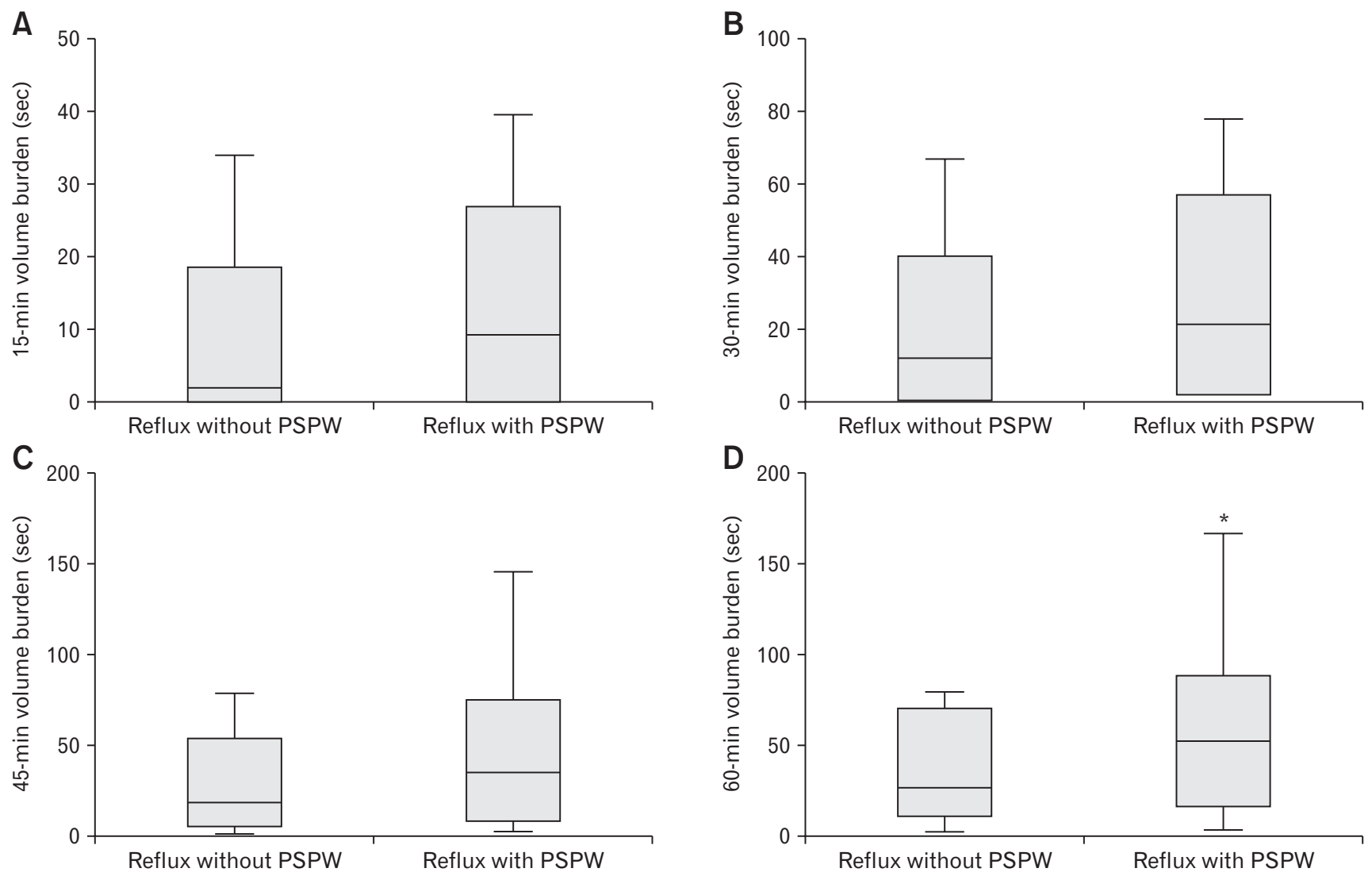

Figure 1. Volume burden between non-post-reflux swallow-induced peristaltic wave (PSPW) group and PSPW group. (A) Fifteen-minute volume burden between the 2 groups $(2.10[0.00,18.90]$ seconds vs $9.35[0.00,27.18]$ seconds, $P=0.936)$. (B) Thirty-minute volume burden between the 2 groups $(12.40[1.00,40.50]$ seconds vs $21.65[4.65,57.10]$ seconds, $P=0.361)$. (C) Forty-five-minute volume burden between the 2 groups (19.00 [6.80, 54.40] seconds vs $35.80[9.70,75.75]$ seconds, $P=0.169)$. (D) Sixty-minute volume burden between the 2 groups $(27.60$ $[12.10,71.00]$ seconds vs $42.15[17.35,89.08]$ seconds, $P=0.027) .{ }^{*} P<0.05$.

mucosa to pre-reflux conditions. Induced by stretch receptors in the esophageal, volume clearance removes almost $90 \%$ of the refluxate via a secondary peristaltic wave. Chemical clearance consists of a swallow-induced peristaltic wave which is elicited by an esophagosalivary vagal reflex and delivers salivary bicarbonate and epidermal growth factor to the distal esophageal, thus raising the $\mathrm{pH}$ level and helping to repair reflux-induced mucosal damage. ${ }^{10}$ Contributing to luminal clearance are gravity and peristalsis for volume clearance, and secretions from salivary and esophageal submucosal glands for acid clearance. ${ }^{11}$ One of defense mechanisms is primary peristalsis which is also known as PSPW. PSPW is a novel impedance parameter used to evaluate esophageal chemical clearance. ${ }^{12}$ As the human esophageal mucosa is very sensitive to continuous exposure to acidic and weakly acidic solutions, it has been proved that impaired chemical clearance or hypersensitivity of the esophagus are associated with decreased PSPW index values. ${ }^{3}$ However, few studies have studied the factors triggering out a PSPW. In this study, the characteristics of reflux episodes which induced PSPW were firstly explored.

The proximal extent of reflux episode is considered as an important factor in generating symptoms, which indirectly infers the volume of the reflux. Symptomatic reflux episodes had a higher median proximal extent than asymptomatic reflux in patients with GERD, which indicates the proximal extent of the refluxate is closely associated with perception of reflux symptoms. ${ }^{13}$ While the volume of reflux is related to total amount of concentrated damaging substances by prolonging distal mucosal exposure or by expanding to more proximal areas. In addition, volume by itself is relevant to the distension of esophagus. In our research, by conducting the ambulatory $\mathrm{pH}$ and impedance monitoring, our data showed that reflux episodes with a PSPW were more likely to be related with a higher volume clearance time and volume burden before episode onset when compared with reflux episodes without a PSPW, in- 

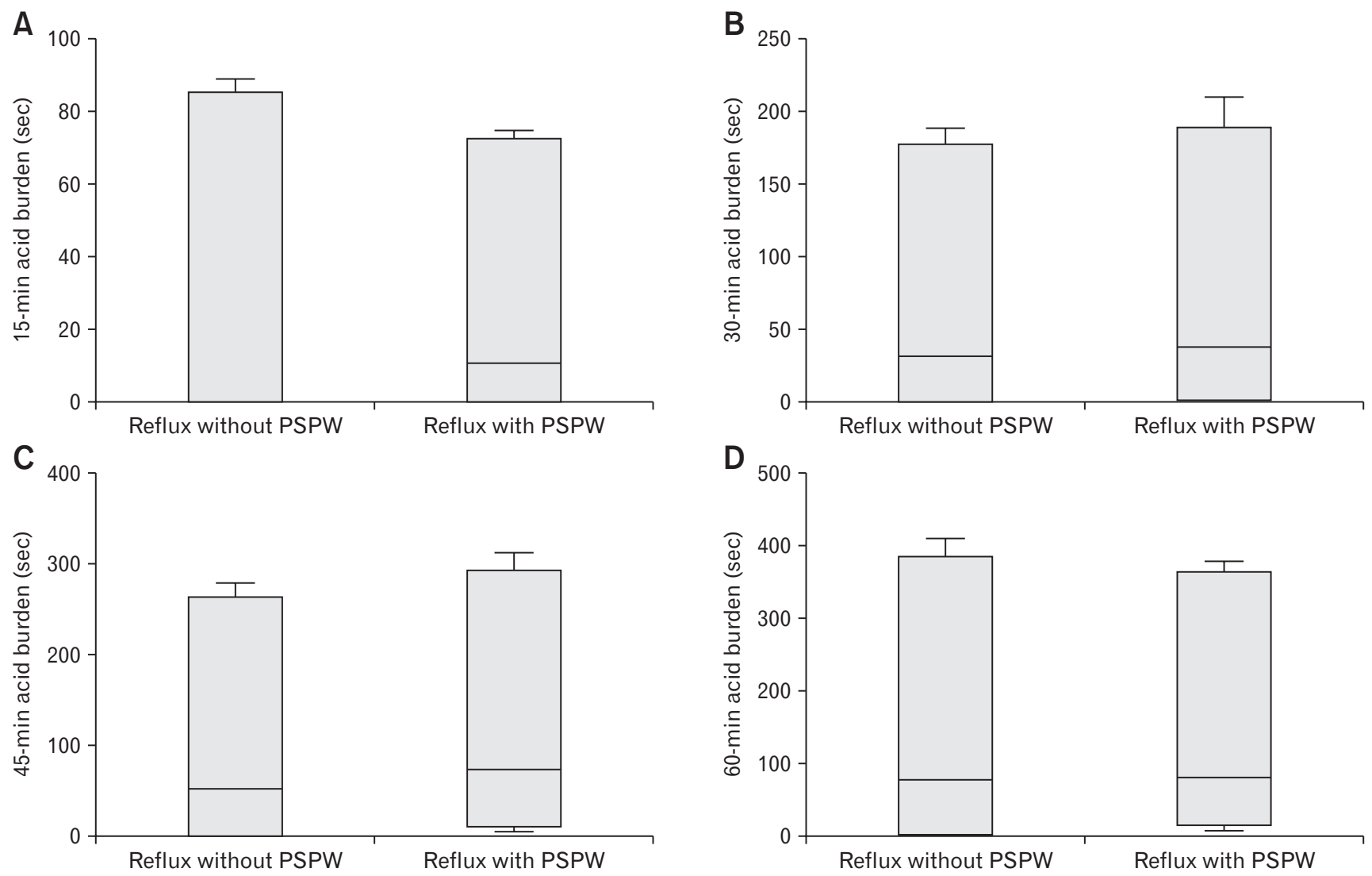

Figure 2. Acid burden between non-post-reflux swallow-induced peristaltic wave (PSPW) group and PSPW group. (A) Fifteen-minute acid burden between the 2 groups $(0.00[0.00,85.7]$ seconds vs $11.40[0.00,72.90]$ seconds, $P=0.058)$. (B) Thirty-minute acid burden between the 2 groups $(32.30[0.00,178.30]$ seconds vs 38.10 [3.10, 190.10] seconds, $P=0.798)$. (C) Forty-five-minute acid burden between the 2 groups $(55.60$ $[0.00,264.80]$ seconds vs $71.10[9.03,290.98]$ seconds, $P=0.670)$. (D) Sixty-minute acid burden between the 2 groups (80.40 [3.40, 386.30$]$ seconds vs $84.05[18.08,365.65]$ seconds, $P=0.693)$.

Table 2. Characteristics of the Study Population

\begin{tabular}{lccc}
\hline \multicolumn{1}{c}{ Characteristics } & Reflux with & Reflux without PSPW & $P$-value \\
& PSPW & $3.70(1.00,6.80)$ & 0.016 \\
Proximal extent (sec) & $5.00(1.40,9.15)$ & $9.00(7.00,17.00)$ & 0.021 \\
Proximal extent $(\mathrm{cm})$ & $17.00(9.00,17.00)$ & $3.40(1.72,10.00)$ & 0.415 \\
Ascending velocity $(\mathrm{cm} / \mathrm{sec})$ & $2.82(1.42,9.86)$ & $2.90(1.95,5.15)$ & 0.323 \\
Nadir pH & $3.10(2.03,5.58)$ & $1.70(0.40,3.95)$ & 0.883 \\
pH drop & $1.65(0.50,3.68)$ & $11.50(6.65,16.70)$ & 0.029 \\
Volume clearance time (sec) & $13.15(8.93,19.65)$ & $4.55(0.00,10.35)$ & 0.273 \\
Acid clearance time (sec) & $4.45(0.00,11.30)$ & $0.44(0.00,0.81)$ & 0.302 \\
Percentage acidic $(\%)$ & $0.22(0.00,0.77)$ & & \\
\hline
\end{tabular}

PSPW, post-reflux swallow-induced peristaltic wave.

$P<0.05$ is considered to indicate a statistically significant difference.

Data are presented as median (interquartile range).

dicating reflux episode with larger volume of the refluxate and a higher volume burden preceding the onset of reflux are more likely to induce a PSPW. We also suggest that when there are highly con- centrated damages in the esophagus or maybe when the esophagus distend to a certain level, a PSPW will been initiated to prevent the mucosa from sustaining damage. Moreover, symptomatic reflux 
episodes with a better perception may also be responsible for producing a PSPW which will be further studied in our center.

Clearance of the esophagus begins with peristalsis, which removes most of the reflux, and then neutralizes acid by swallowed saliva. ${ }^{14}$ Volume clearance time depends on the effectiveness of esophageal peristalsis. The intraluminal distension is the principal stimulus for upper esophageal clearance. In this study, it is found out that reflux episodes with a PSPW were more related with a higher volume clearance time which indicates that intraluminal distension in the upper esophageal and depression of esophageal peristalsis may be responsible for triggering a PSPW after the reflux. When the ability of volume clearance is impaired, the efficiency of chemical clearance will probably be enhanced relatively.

In addition, no significant differences were found between the 2 groups in nadir $\mathrm{pH}, \mathrm{pH}$ drop, acid clearance time, percentage acidic, or acid burden which revealed that acid concentration or acid burden were not responsible for PSPW generation. We indicate that GERD patients with higher PSPW index may not benefit well from proton pump inhibitors, while prokinetic agents may help to relieve symptoms.

\section{Conclusion}

PSPW is a self-protection mechanism after a reflux. Proximal reflux episodes and large volume burden are more likely to induce a PSPW. Depression of esophageal peristalsis and motility may play an important role in PSPW generation. However, the role of acid seems to be less important in reflux episodes which could induce a PSPW. The limitations of our research are that the samples were small and the main symptom of the GERD patient enrolled was cough. Future researches need to be carried out on larger samples and patients with typical symptoms to investigate the determinants of PSPW in reflux episodes of GERD which may help in finding out a new therapeutic strategy and understanding the mechanism of GERD.

\section{Financial support: None.}

\section{Conflicts of interest: None.}

Author contributions: Hairong $\mathrm{Xu}$, Bixing Ye, Lin Lin, and Linqin Jiang conceived and designed the experiments; Hairong $\mathrm{Xu}$, Bixing Ye, Yu Ding, and Meifeng Wang performed the experiments; Hairong Xu, Bixing Ye, and Yu Ding analyzed the data; Hairong $\mathrm{Xu}$, Bixing Ye, and Liuqin Jiang contributed reagents/ materials/analysis tools; and Hairong Xu and Liuqin Jiang wrote the manuscript.

\section{References}

1. Vakil N, van Zanten SV, Kahrilas P, Dent J, Jones R; Global Consensus Group. The Montreal definition and classification of gastroesophageal reflux disease: a global evidence-based consensus. Am J Gastroenterol 2006;101:1900-1920.

2. Roman S, Gyawali CP, Savarino E, et al. Ambulatory reflux monitoring for diagnosis of gastro-esophageal reflux disease: update of the porto consensus and recommendations from an international consensus group. Neurogastroenterol Motil 2017;29:1-15.

3. Frazzoni L, Frazzoni M, de Bortoli N, et al. Postreflux swallow-induced peristaltic wave index and nocturnal baseline impedance can link PPIresponsive heartburn to reflux better than acid exposure time. Neurogastroenterol Motil 2017;29:e13116.

4. Frazzoni M, Bertani H, Manta R, et al. Impairment of chemical clearance is relevant to the pathogenesis of refractory reflux oesophagitis. Dig Liver Dis 2014;46:596-602.

5. Shaw MJ, Talley NJ, Beebe TJ, et al. Initial validation of a diagnostic questionnaire for gastroesophageal reflux disease. Am J Gastroenterol 2001;96:52-57.

6. Zhong C, Duan L, Wang K, et al. Esophageal intraluminal baseline impedance is associated with severity of acid reflux and epithelial structural abnormalities in patients with gastroesophageal reflux disease. J Gastroenterol 2013;48:601-610.

7. Simrén M, Silny J, Holloway R, Tack J, Janssens J, Sifrim D. Relevance of ineffective oesophageal motility during oesophageal acid clearance. Gut 2003;52:784-790.

8. Bredenoord AJ, Weusten BL, Curvers WL, Timmer R, Smout AJ. Determinants of perception of heartburn and regurgitation. Gut 2006;55:313-318.

9. Yoshida N, Yoshikawa T. Defense mechanism of the esophageal mucosa and esophageal inflammation. J Gastroenterol 2003;38(suppl 15):31-34.

10. Frazzoni M, de Bortoli N, Frazzoni L, et al. The added diagnostic value of postreflux swallow-induced peristaltic wave index and nocturnal baseline impedance in refractory reflux disease studied with on-therapy impedance-pH monitoring. Neurogastroenterol Motil 2017;29:e12947.

11. Orlando RC. The integrity of the esophageal mucosa. Balance between offensive and defensive mechanisms. Best Pract Res Clin Gastroenterol 2010;24:873-882.

12. Cho YK, Lee JS, Lee TH, et al. The relationship of the post-reflux swallow-induced peristaltic wave index and esophageal baseline impedance with gastroesophageal reflux disease symptoms. J Neurogastroenterol Motil 2017;23:237-244.

13. Weusten BL, Akkermans LM, vanBerge-Henegouwen GP, Smout AJ. Symptom perception in gastroesophageal reflux disease is dependent on spatiotemporal reflux characteristics. Gastroenterology 1995;108:17391744

14. Helm JF, Dodds WJ, Pelc LR, Palmer DW, Hogan WJ, Teeter BC. Effect of esophageal emptying and saliva on clearance of acid from the esophagus. N Engl J Med 1984;310:284-288. 